OPEN ACCESS

Edited by:

Tao Jiang,

Shanghai Pulmonary Hospital,

China

Reviewed by:

Yuan Wan,

Binghamton University,

United States

Chao Wang,

Southeast University, China

*Correspondence: Jun Zhang junzhang10977@sjtu.edu.cn

${ }^{+}$These authors have contributed equally to this work and share first authorship

Specialty section: This article was submitted to

Cancer Immunity and Immunotherapy,

a section of the journa

Frontiers in Oncology

Received: 17 March 2021 Accepted: 06 April 2021

Published: 22 April 2021

Citation:

Yuan H, Ji J, Shi M, Shi Y, Liu J, Wu J, Yang C, Xi W, Li Q, Zhu W, Li J,

Gong $X$ and Zhang J (2021)

Characteristics of Pan-Cancer

Patients With Ultrahigh Tumor

Mutation Burden.

Front. Oncol. 11:682017.

doi: 10.3389/fonc.2021.682017

\section{Characteristics of Pan-Cancer Patients With Ultrahigh Tumor Mutation Burden}

\author{
Hong Yuan ${ }^{1+}$, Jun $\mathrm{Ji}^{2 \dagger}$, Min Shi ${ }^{1}$, Yan Shi ${ }^{1}$, Jing Liu ${ }^{1}$, Junwei Wu ${ }^{1}$, Chen Yang ${ }^{1}$, \\ Wenqi $X i^{1}$, Qingyuan $L i^{3}$, Wei Zhu ${ }^{3}$, Jingjie $L i^{3}$, Xiaoli Gong ${ }^{3}$ and Jun Zhang ${ }^{1,4^{*}}$ \\ 1 Department of Oncology, Ruijin Hospital, Shanghai Jiao Tong University School of Medicine, Shanghai, China, \\ 2 Shanghai Institute of Digestive Surgery, Ruijin Hospital, Shanghai Jiao Tong University School of Medicine, Shanghai, China, \\ ${ }^{3}$ Genecast Biotechnology Co., Ltd, Wuxi, China, ${ }^{4}$ State Key Laboratory of Oncogenes and Related Genes, Shanghai Jiao \\ Tong University, Shanghai, China
}

Background: Tumor mutation burden has been proven to be a good predictor for the efficacy of immunotherapy, especially in patients with hypermutation. However, most research focused on the analysis of hypermutation in individual tumors, and there is a lack of integrated research on the hypermutation across different cancers. This study aimed to characterize hypermutated patients to distinguish between these patients and nonhypermutated patients.

Methods: A total of 5,980 tumor samples involving 23 types of solid tumors from the inhouse database were included in the study. Based on the cutoff value of tumor mutation burden (TMB), all samples were divided into hypermutated or non-hypermutated groups. Microsatellite instability status, PD-L1 expression and other mutation-related indicators were analyzed.

Results: Among the 5,980 tumor samples, 1,164 were selected as samples with hypermutation. Compared with the non-hypermutated group, a significant increase in the mutation rates of DNA mismatch repair genes and polymerase genes was detected in the hypermutated group, and there was an overlap between high TMB and high microsatellite instability or high PD-L1. In addition, we found that EGFR, KRAS and PIK3CA had a high frequency of both single nucleotide variation and copy number variation mutations. These identified mutant genes were enriched in the oncogenic signaling pathway and the DNA damage repair pathway. At the same time, the somatic cell characteristics and distribution of the two groups were significantly different.

Conclusions: This study identified genetic and phenotypic characteristics of hypermutated tumors and demonstrated that DNA damage repair is critically involved in hypermutation.

Keywords: pan-cancer, hypermutation, tumor mutation burden, mismatch repair, polymerase 


\section{INTRODUCTION}

The fact that many different cancers share common genomic characteristics (1) and respond well to relevant inhibitors has led researchers to perform integrated studies involving multiple types of cancers. Comparison of tumor types analyzed by The Cancer Genome Atlas (TCGA) through the Pan-Cancer Atlas can further supplement and summarize the completed TCGA results (2). The integration of these data sets provides a comprehensive picture of somatic mutations $(3,4)$, copy number changes $(5,6)$, mutational signatures (7), and other genetic variations in tumors, furthering the understanding of cancer mechanisms.

Tumor mutation burden (TMB) is defined as the total number of somatic gene coding errors, base substitutions, and gene insertions or deletions detected per million bases (8). The number of somatic mutations in different types of cancers ranges from $0.01 \mathrm{mut} / \mathrm{Mb}$ to more than $400 \mathrm{mut} / \mathrm{Mb}$. Tumor antigenicity increases with increased TMB and is a prerequisite for PD1/PDL1 antibody efficacy. In recent years, TMB has been proven to be a good predictor for the efficacy of immunotherapy in multiple clinical trials $(9,10)$. Retrospective analysis of the CheckMate 568 clinical trial revealed that among patients with advanced/metastatic NSCLC, those with a TMB of $10 \mathrm{mut} / \mathrm{Mb}$ or higher had higher objective response and progression-free survival rates than those with a TMB of less than $10 \mathrm{mut} / \mathrm{Mb}$ (11). Similar results were observed in the KEYNOTE-028 trial (12).

Hypermutation refers to a cellular mechanism that causes the genome to be mutated at a frequency at least 100,000 to millions of times higher than the background mutation rate. It mainly involves point mutations (single base substitution), as well as occasional base insertion or deletion. Many types of cancers, such as colorectal cancer (13) and gastrointestinal cancer (14,) are classified into two molecular pathological groups: hypermutation and non-hypermutation. Recently, several longitudinal observational studies conducted comparisons of glioma and prostate cancer before and after treatment and found hypermutation differences in the genomes of patients, in particular when the tumor recurs (15-17). In the case of hypermutation, an increasing number of mutations in hypermutant cells may result in decreased fitness, rendering the cells less aggressive and more susceptible to treatment (18). Therefore, hypermutation plays an essential role in tumor occurrence and progression and can improve therapeutic efficacy. However, to date, most research has focused on the analysis of hypermutation in individual tumors, and there is a lack of integrated research on the hypermutation of different cancers.

Here, we performed a comprehensive pan-cancer classification of 5,980 tumor samples involving 23 types of solid tumors from the in-house database (Genecast Biotechnology Co., Ltd). This study aimed to identify the differences in characteristics of the genome mutation profile between patients with hypermutation and those with nonhypermutation (low group). The findings may have significance in guiding clinical practice.

\section{MATERIALS AND METHODS}

\section{Genomic and Clinical Data}

Genomic and clinical data from 23 different types of solid tumors were gathered from the in-house database (Genecast Biotechnology Co., Ltd). The in-house database was built based on the information collected from clinical samples that was sequenced by a customized 543-gene panel, which covered 1.7 $\mathrm{Mb}$ of the genome. The filtering criteria for the samples used in this study were as follows: 1). Samples that were sequenced from January 1, 2019 to December 31, 2019; 2). Samples that were tested by a 543 -gene panel; 3). Tissue samples; 4 ). Patients who aged $>25$ years old; 5) samples that were collected at the earliest time. The exclusion criteria were as follows: 1 ). Samples with $\mathrm{TMB}=0 ; 2$ ). Tumors with $<20$ samples; 3 ). Metastatic samples. The following types of solid tumors were included in the study: non-small cell lung cancer (adenocarcinoma, LUAD, $\mathrm{n}=2384$; squamous cell carcinoma, LUSC, $\mathrm{n}=456$; others, NSCL, $\mathrm{n}=554$ ), stomach cancer (STAD, $n=534)$, colon cancer (COAD, $n=476$ ), rectal cancer (READ, $\mathrm{n}=344$ ), esophageal carcinoma (ESCA, $\mathrm{n}=184$ ), liver hepatocellular carcinoma (LIHC, $\mathrm{n}=162$ ), cholangiocarcinoma ( $\mathrm{CHOL}, \mathrm{n}=123$ ), pancreatic cancer (PAAD, $\mathrm{n}=120$ ), breast cancer (BRCA, $\mathrm{n}=111$ ), head and neck squamous cell carcinoma (HNSC, $\mathrm{n}=95$ ), small cell lung cancer ( $\mathrm{SCL}, \mathrm{n}=80$ ), ovarian cancer (OV, $\mathrm{n}=60$ ), cervical squamous cell carcinoma (CESC, $\mathrm{n}=47$ ), glioblastomas ( $\mathrm{GBM}, \mathrm{n}=47$ ), nasopharyngeal cancer (NASO, $\mathrm{n}=38$ ), skin cutaneous melanoma (SKCM, $\mathrm{n}=35$ ), bladder cancer (BLCA, $\mathrm{n}=29$ ), kidney cancer (LICH, $\mathrm{n}=28$ ), soft tissue sarcoma (SARC, $\mathrm{n}=28$ ), uterine corpus endometrial carcinoma (UCEC, $\mathrm{n}=25$ ), and gastrointestinal stromal cancer (GIST, $\mathrm{n}=20$ ). The cutoff value for hypermutation (8.561943) was determined by the segmented linear regression analysis in $\mathrm{R}$ language $(19,20)$. Among 5,980 Chinese patients, 1,164 (19.5\%) had a significantly higher TMB than the others and were identified as patients with hypermutation.

\section{Identification of Mismatch Repair and Polymerase Gene Mutations}

After analyzing the population frequency in the database, as well as the cosmic database and dbSNP database, we screened for nonsynonymous mutations in the exon region or cleavage region of DNA mismatch repair (MMR) genes (MSH2, MLH1, MSH6, and PMS2) and polymerase genes (POL; POLE and POLD1). Manual review was performed to determine the final mutation set. Samples containing more than one mutation in the 6 genes were identified as MMR/POL mutation samples. The Wilcoxon test was used to compare the distribution of TMB between the mutation (MUT) group and wild-type (WT) group, while the difference in the proportion of samples with MUT or WT between the Hypermutation group and Low group was analyzed by using Fisher's test.

\section{Analysis of Microsatellite Instability (MSI)}

For each microsatellite locus, all spanning reads (covering at least $2 \mathrm{bp}$ in both the 5' and 3' directions) were extracted from the realigned BAM file. Following deduplication, the length of the 
mononucleotide repeat in each deduped alignment was counted and tallied by length. The baseline reference value was calculated by using 30 normal blood samples and was used to assess the instability of microsatellite loci. Finally, the fraction of unstable loci out of the total number of loci analyzed was calculated for each experimental sample. Based on the fraction value, samples were classified into the MSI-H group and MSS/MSI-L group. A fraction value of 0.3 was set as the cutoff value for defining an unstable locus as an MSI-positive locus. The Wilcoxon test was used to compare the distribution of TMB between the MSI-H group and MSS/MSI-L group, while the difference in the proportion of samples with MSI-H or MSS/MSI-L between the Hypermutation group and Low group was analyzed by using Fisher's test.

\section{Detection of PD-L1 Expression}

The expression of PD-L1 on the surface of tumor cells (TCs) and tumor-infiltrating immune cells (ICs) was assessed by IHC staining using anti-PD-L1 (SP142) rabbit monoclonal primary antibody (Roche, Indianapolis, IL, USA). PD-L1 expression was described as a continuous variable based on the percentage of tumor cells with a certain staining intensity (21). Samples were also classified into the negative $(\mathrm{N})$, low-positive (positive $1, \mathrm{P} 1$ ), medium-positive (positive 2, P2), and high-positive (positive 3 , P3) groups according to the expression level of PD-L1. The Wilcoxon or Kruskal-Wallis test was used to compare the distribution of TMB in the high and low PD-L1 groups, while the difference in PD-L1 expression between the Hypermutation group and the Low group was analyzed by using Fisher's test.

\section{Identification of Single Nucleotide Variation (SNV)}

Sequencing reads were processed through an in-house pipeline. The pipeline included Trimmomatic (v.0.39) for read adapter trimming and quality filtering, BWA (v.0.7.17) for mapping reads to the hg19 reference genome, the Picard toolkit (v.2.1.0) for sorting and making duplicates, and the Genome Analysis ToolKit (v.3.7) for read realignment. VarDict (v.1.5.1) was introduced for SNV calling, and compound heterozygous mutations were merged with FreeBayes (v.1.2.0). The generated candidate mutations were annotated using the ANNOVAR software tool and then filtered by using the ExAC, COSMIC, and dbSNP databases. Manual curation was performed to generate the final somatic SNV/InDel data set. The differences between the two groups of variation were evaluated by Fisher's test.

\section{Identification of Copy Number Variation (CNV)}

The GC content, target region length, and read count were corrected. Thereafter, the copy number and gene specificity score (GCS) was calculated using 30 normal blood samples as a control. GCS represents the degree of gene level difference between the tested sample and control. CNV was determined by a joint statistical significance test on GCS and the absolute value of the copy number.

\section{Pathway and Mutational Signature Analysis}

We identified genetic mutations in 10 major cancer pathways and 8 repair pathways in the samples, counted the number of mutations in each pathway for each cancer population and calculated the mutation frequency of each cancer population. The mutational signature was determined based on these somatic SNVs/InDels using maftools (v.2.4.10). The Wilcoxon test was used to compare the distribution and difference of the somatic signature among or between the Hypermutant and Low groups.

\section{RESULTS}

\section{TMB Screening-Based Detection of Hypermutation}

As shown in Figure 1A, 1,164 patients from a cohort of 5,980 patients with pan-cancer in the in-house dataset were selected as those with hypermutation based on the calculated cutoff value of TMB. The median value of the calculated TMB for each group is shown in Figure 1B. Notably, the TMB values of GBM and UCEC were much higher in the Hypermutation group (Figure 1C). The age was older and male proportion was higher in the Hypermutation group than in the Low group $(\mathrm{p}<0.05)$ (Table S1).

\section{MSI Status and PD-L1 Expression of Patients With Hypermutation}

We next evaluated the impact of TMB on MSI and PD-L1 expression using statistical methods to identify events associated with TMB in solid tumors. MSI, especially high MSI (MSI-H), is closely associated with the occurrence and progression of many tumors. In all samples, the MSI-H samples had a significantly higher TMB than the MSS/MSI-L samples (Figure 2A). No difference in TMB was observed between the MSI-H and MSS/ MSI-L groups due to the low frequency of MSI in LUSC, HNSC, LICH, PAAD, SKCM, LUAD and other solid tumors. In contrast, there were significant differences in TMB values among UCEC $(P$ $<0.05)$, COAD $(P<0.001)$, READ $(P<0.001)$, NSCL $(P<0.01)$, $\operatorname{STAD}(P<0.001)$, CHOL $(P<0.01)$ and NASO $(P<0.05)$, indicating that TMB was elevated in MSI-H samples (Figure 2B). Moreover, analysis of Hypermutation and Low samples revealed that Hypermutation samples were more prone to MSI$\mathrm{H}$ (Figures 2C, D).

Similar to the analysis of MSI-H characteristics, studies on PD-L1 expression showed that the P2/P3 group displayed a significantly higher overall TMB than the $\mathrm{N}$ group, albeit in only six types of tumors, including COAD $(P<0.01)$, READ $(P<0.05)$, NSCL $(P<0.01)$, STAD $(P<0.01)$, SARC $(P<0.05)$, and LUAD $(P$ $<0.0001$ ) (Figures 3A, B). In addition, a comparison of the difference between Hypermutation and Low samples suggested that there was a correlation between high TMB and high expression of PD-L1 (Figures 3C, D). 
A
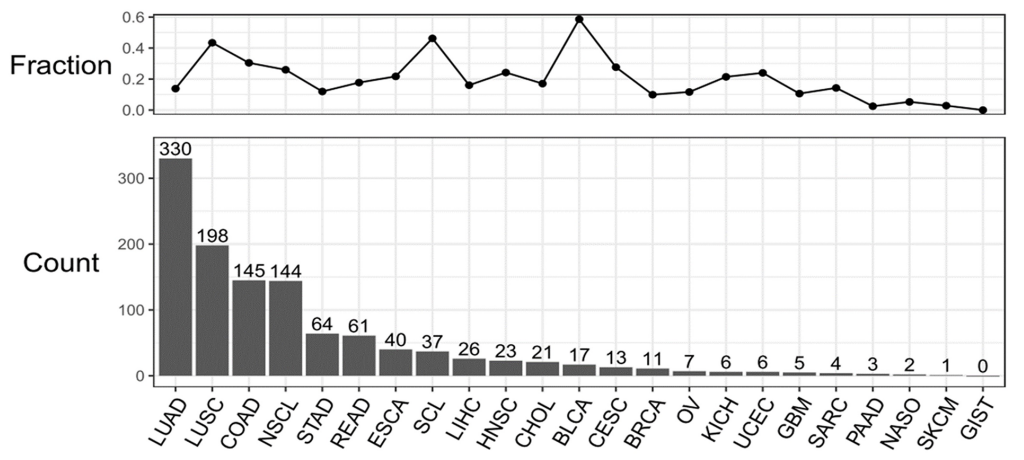

B

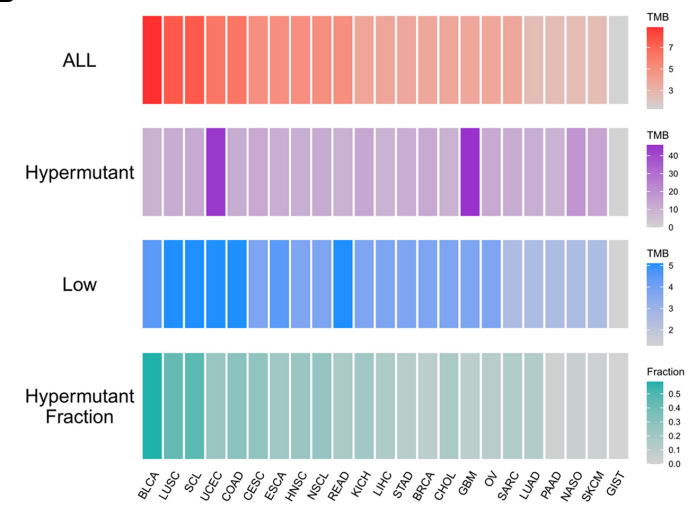

C

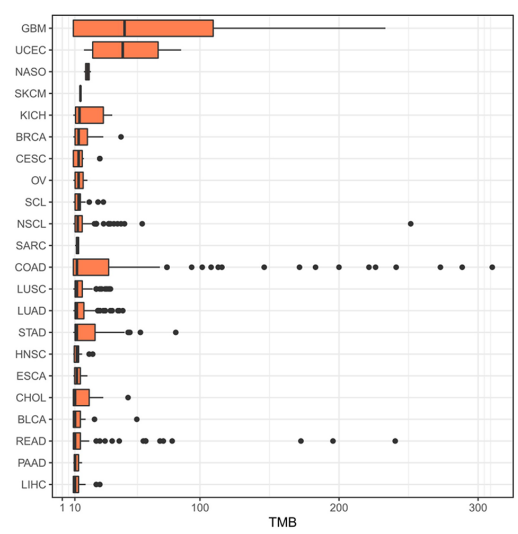

FIGURE 1 | The distribution of cancer types. (A) Histogram of the number of samples with hypermutation per cancer type. The numbers were sorted from highest to lowest. The line graph shows the proportion of the number of samples with hypermutation to the total number of samples for each cancer type. (B) The median value of TMB in the three sample groups (ALL, Hypermutation, and Low) for each cancer type and the number of Hypermutation samples for each cancer type as a proportion of the total number of samples for that cancer type. (C) The distribution of TMB in samples with hypermutation for each cancer type.

\section{Mutational Characteristics of Patients With Hypermutation}

While MSI is caused by a defect in MMR genes, POLE or POLD1 mutations serve as immunotherapeutic indicators of all types of tumors except for those with MSI-H. Therefore, we first examined the distribution of TMB in the MUT group and WT group at the global and carcinoma-specific hypermutation level. As shown in Figures 4A, B, samples with MMR and/or POL mutations had a higher TMB than the MUT group. The TMB values of COAD, GBM and UCEC were higher than those of other cancer types, and there were significant differences between COAD and UCEC in the MUT and WT groups $(P<0.0001)$. Combined with the data from the Low group, a redescription of the mutation landscape for the two types of samples revealed that Hypermutation samples harbored more MMR/POL mutations than the MUT group (Figures 4C, D).

We next investigated whether the TMB level affects tumor mutation and CNV burden by quantifying the mutation rate and percentage of CNV in each group. Table 1 lists CNVs with significant differences between the Hypermutation group and the Low group. Three genes (EGFR, KRAS and PIK3CA) in the top 10 list of mutated genes were identified as having significantly differential CNVs (Figure 5). In addition, TP53 was found to be the gene with the highest mutation frequency, with missense mutation as the main mutation type.

Statistical analysis of genetic mutations in 10 major cancer pathways and 8 repair pathways in the two types of samples revealed that mutations in the Hypermutation group were mainly enriched in p53 and RTK-RAS cancer-related pathways as well as the homologous recombination and MMR pathways. The mutation frequency of each pathway differed between the two samples (Figure 6). To better understand pathways globally dysregulated in the setting of TMB, we further performed a somatic signature analysis in the Hypermutation and Low groups. As shown in Figure 7, a total of five signatures, including defective DNA MMR and defects in polymerase POLE, displayed significant differences between the two groups $(P<0.0001)$.

\section{DISCUSSION}

In this study, we delineated the distribution of cancer types in patients with hypermutation and identified an association 
A

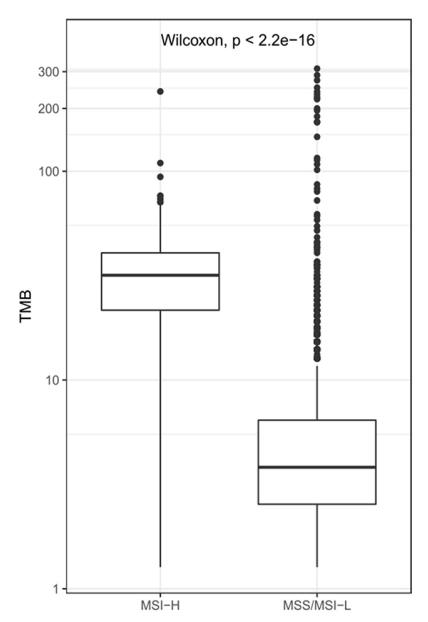

B

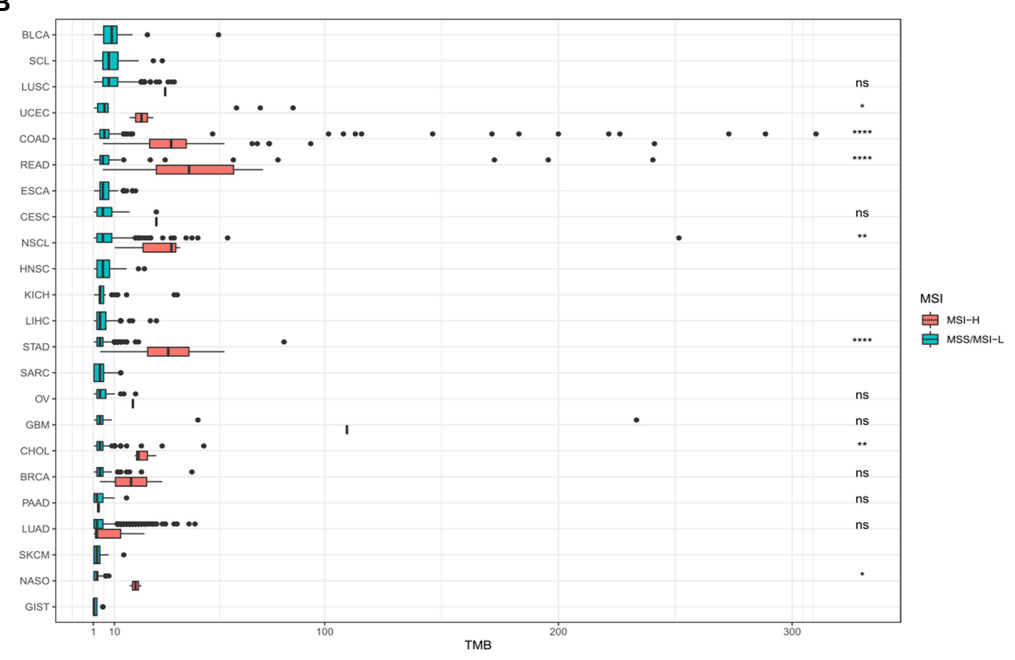

C

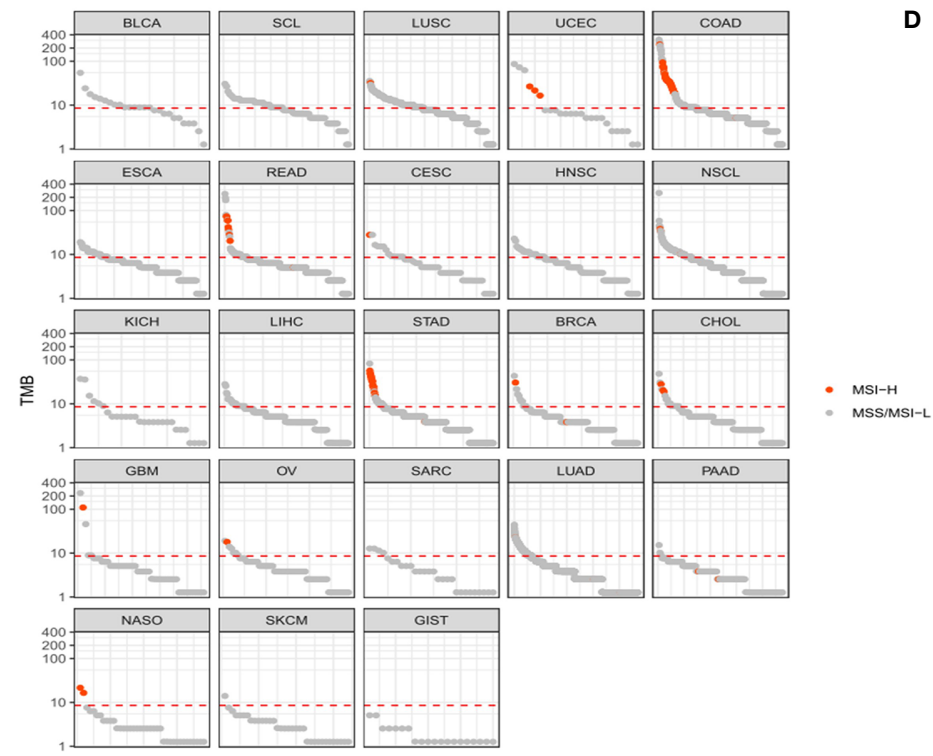

D

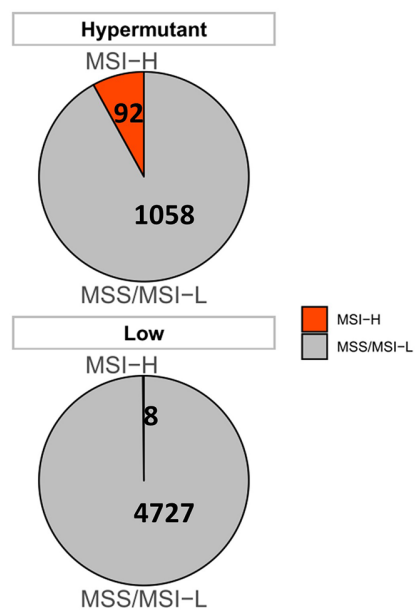

$P$ value $<2.2 e-16$

FIGURE 2 | (A) Comparison of TMB distribution between MSI-H and MSS/MSI-L groups. (B) TMB distribution in different cancer types grouped according to high and low MSI. (C) TMB value of each sample in each cancer type. Orange and gray represent MSI-H samples and MSS/MSI-L samples, respectively. (D) The number and proportion of MSI-H and MSS/MSI-L samples in Hypermutation and Low groups. $P<0.05$ was considered a significant difference, ${ }^{\star} P<0.05$, ${ }^{\star \star} P<0.01$, ${ }^{\star \star \star \star} P<0.0001$, NS, Not Significant.

between hypermutation and MSI status, PD-L1 expression and MMR/POL gene mutations. This finding is similar to Gong's report, which suggested that POLE mutations and MSI tumors (hypermutation phenotype) may increase the expression of immune checkpoint genes, including PD-1, PD-L1 and CTLA4 (22). Moreover, the present study showed that a comprehensive dissection of high-frequency CNVs, related pathways and somatic signatures, as well as the identification of highfrequency SNVs, are required to identify hypermutation cases with unique characteristics.

Known immune efficacy markers can be roughly divided into two categories: the first is related to tumor neoantigen load, including molecular markers such as MSI or TMB elevation, while the second is related to the tumor $\mathrm{T}$ cell inflammatory microenvironment, including core gene markers for PD-L1 protein expression, tumor lymphocyte infiltration and CNV (23). These two types of markers reflect the overall picture of tumor immune efficacy. A combination of two or more methods to determine the immune status of the tumor microenvironment is an effective and universal approach for predicting the efficacy of immune checkpoint inhibitors. In investigating the relationship between MSI or PD-L1 and TMB, we emphasized that the effect of MSI or PD-L1 on TMB mutation rates may vary with tumor type and may be influenced by other endogenous and 


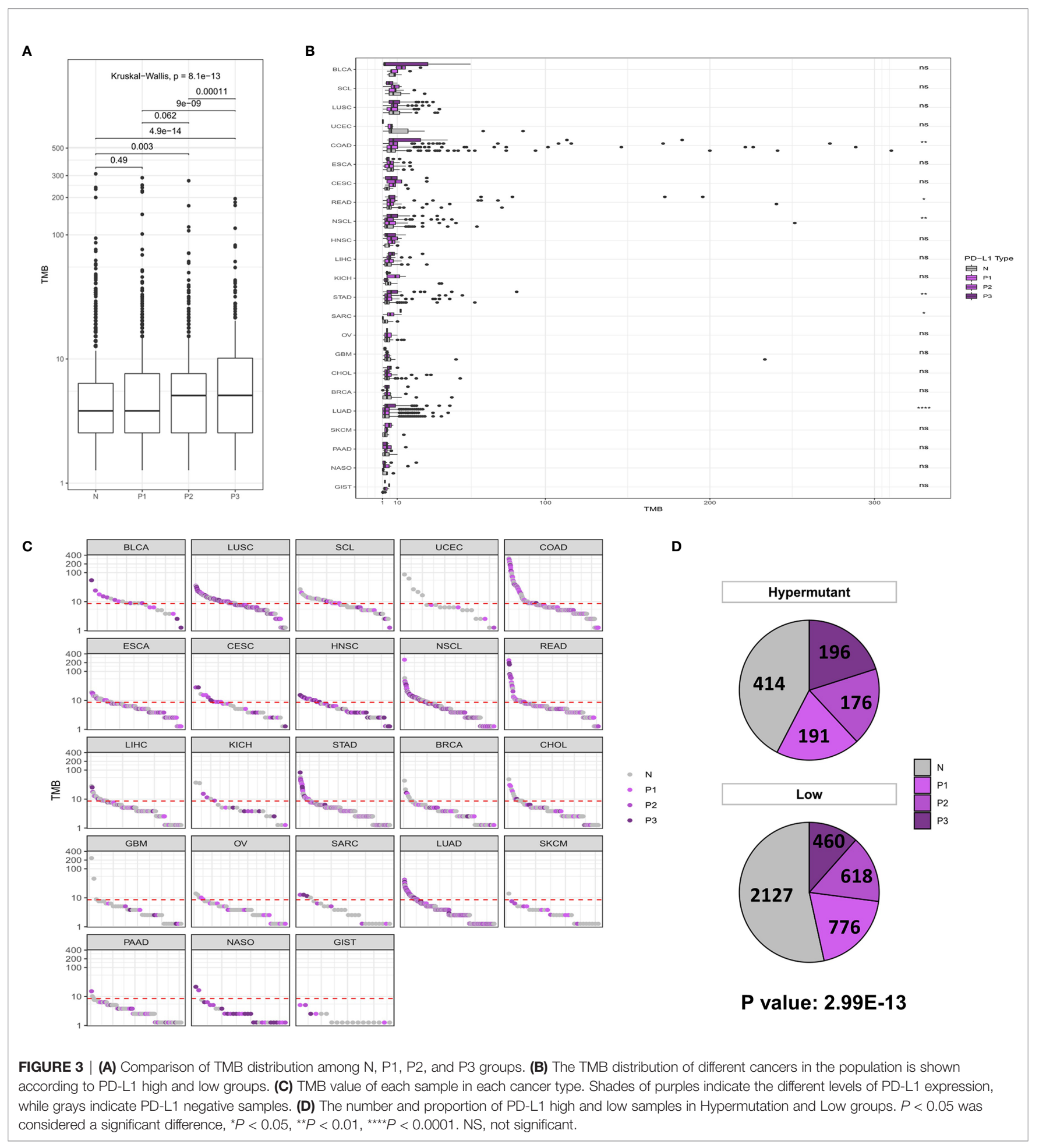

exogenous tumor factors, such as tumor-infiltrating lymphocytes and microbial flora. It has been documented that a high proportion of hypermutation cooccurs with MSI-H or high PDL1 expression in colorectal and ovarian cancers (24-26). Interestingly, MMR/POL mutations have been shown to be associated with higher TMB in pan-cancer patients. MSI is caused by MMR defects due to the inactivation of one of the four main MMR genes, MSH2, MLH1, MSH6, and PMS2, and is characterized by extensive polymorphism in microsatellite sequence length as a result of DNA polymerase slippage (27). Furthermore, studies have shown that tumors with MMR defects can also contain other DNA repair defects, such as POLD or POLE 
A

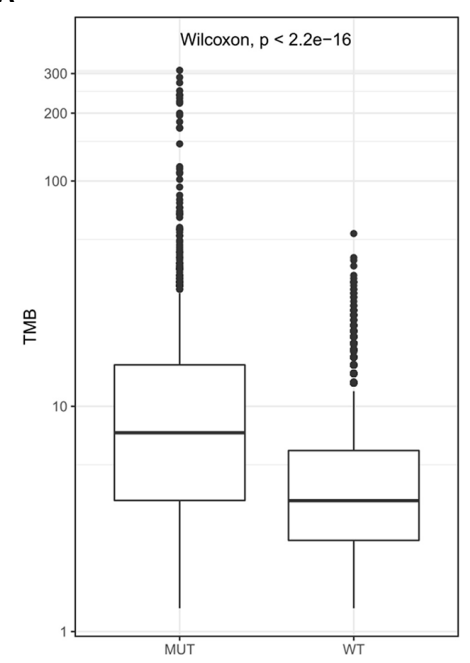

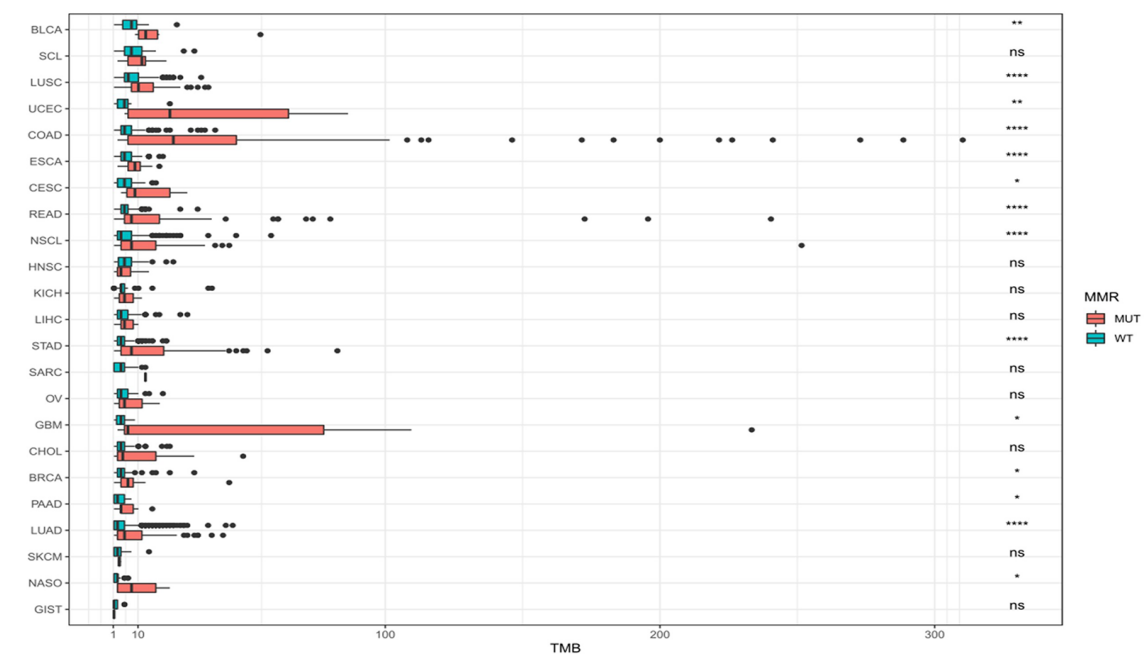

C
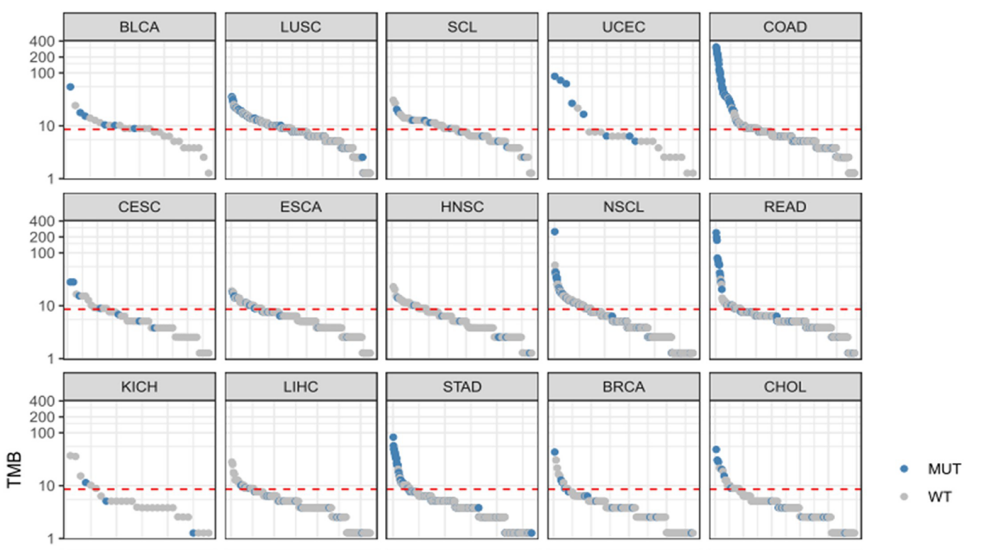

D
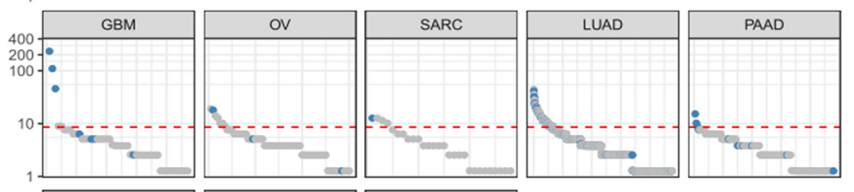

- WT

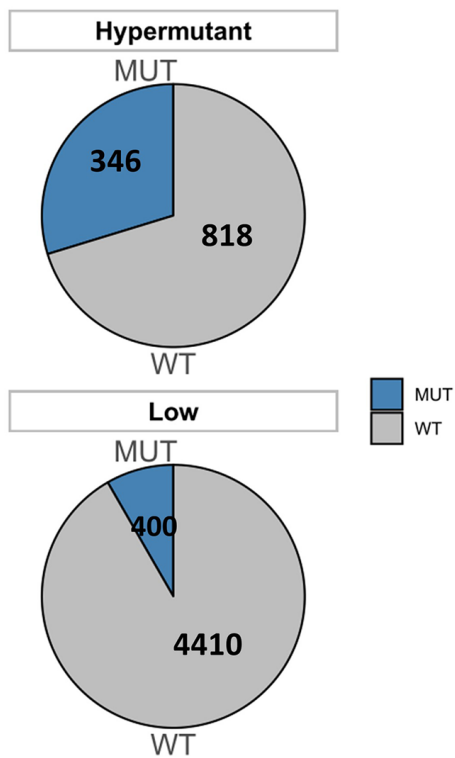

$P$ value $<2.2 \mathrm{e}-16$

FIGURE 4 | (A) Comparison of TMB distribution between the MUT group and WT group. (B) The distribution of TMB in the MUT group and WT group for each cancer type. (C) TMB value of each sample in each cancer type. Blue and gray represent samples in the MUT group and those in WT group, respectively. (D) The number and proportion of MUT and WT samples in Hypermutation and Low groups. $P<0.05$ was considered a significant difference, ${ }^{*} P<0.05$, ${ }^{\star \star} P<0.01$, ${ }^{\star \star \star \star} P<0.0001$. NS, not significant.

mutations, and several immune checkpoint ligands, including PD-1, PD-L1, CTLA-4, LAG-3 and IDO, are also highly expressed in the tumor microenvironment of these patients $(28,29)$. Therefore, MMR and/or POL mutations may underlie the complex interaction between MSI or PD-L1 expression and TMB.

In the present study, we further demonstrated that patients with hypermutation had a much higher frequency of MMR and/or POL mutations than those with non-hypermutation. On the one hand, we observed that among the eight pathways of the DNA damage response system, the homologous recombination and MMR pathways were the most frequently mutated in the tumor samples. Notably, the correlation between MMR and homologous recombination pathways has been reported in colon cancer and rectal cancer (30). On the other hand, we showed in the somatic signature that hypermutant tumors have defects in both MMR genes and the POLE polymerase gene. Similarly, one study looked 
TABLE 1 | The number and proportion of samples with CNV mutation in the Hypermutation and Low groups.

\begin{tabular}{|c|c|c|c|c|c|c|c|c|}
\hline CNV & Class & Total & Var_num & Var_per (\%) & Novar_num & Novar_per (\%) & $P$ value & Odds ratio \\
\hline \multirow[t]{2}{*}{ ALK_GAIN } & Hypermutation & 633 & 5 & 0.79 & 628 & 99.21 & 0.0290771 & 4.916107 \\
\hline & Low & 1857 & 3 & 0.16 & 1854 & 99.84 & & \\
\hline \multirow[t]{2}{*}{ CDK4_GAIN } & Hypermutation & 633 & 31 & 4.90 & 602 & 95.10 & 5.13E-06 & 0.4267569 \\
\hline & Low & 1857 & 200 & 10.77 & 1657 & 89.23 & & \\
\hline \multirow[t]{2}{*}{ EGFR_GAIN } & Hypermutation & 633 & 68 & 10.74 & 565 & 89.26 & 0.001678634 & 0.642541 \\
\hline & Low & 1857 & 293 & 15.78 & 1564 & 84.22 & & \\
\hline \multirow[t]{2}{*}{ KRAS_GAIN } & Hypermutation & 633 & 21 & 3.32 & 612 & 96.68 & 0.02631793 & 0.5784877 \\
\hline & Low & 1857 & 104 & 5.60 & 1753 & 94.40 & & \\
\hline \multirow[t]{2}{*}{ MET_GAIN } & Hypermutation & 633 & 21 & 3.32 & 612 & 96.68 & 0.03308558 & 0.5904969 \\
\hline & Low & 1857 & 102 & 5.49 & 1755 & 94.51 & & \\
\hline \multirow[t]{2}{*}{ PIK3CA_GAIN } & Hypermutation & 633 & 103 & 16.27 & 530 & 83.73 & 5.70E-08 & 2.148361 \\
\hline & Low & 1857 & 154 & 8.29 & 1703 & 91.71 & & \\
\hline
\end{tabular}

Var_num, Number of variation; Var_per, Percentage of variation; Novar_num, Number of no variation; Novar_per, Percentage of no variation; P value and odds ratio were tested using Fisher's analysis.

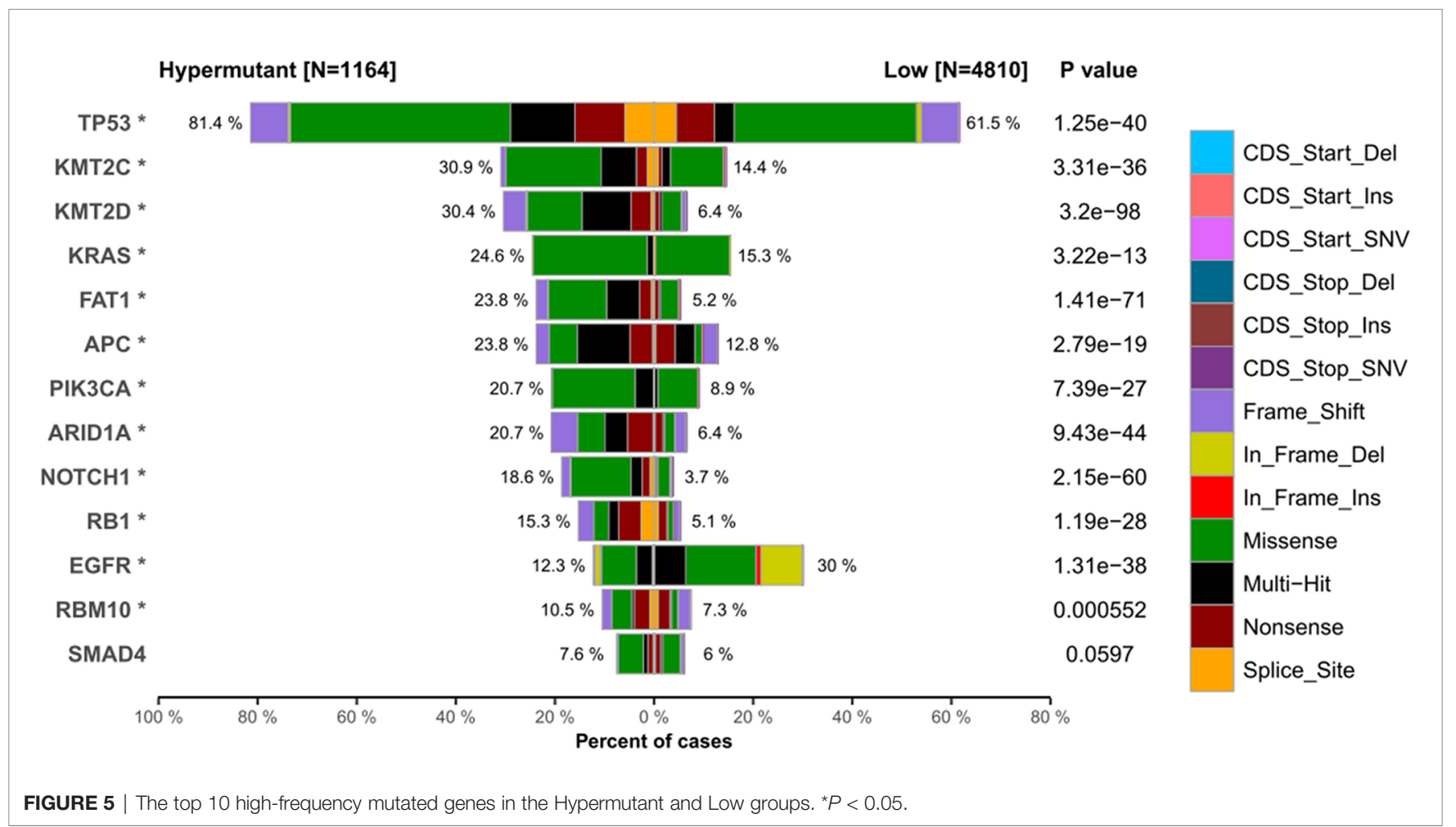

at TCGA PanCancer studies involving 10,967 samples as of November 2018 and found 92 POLE exonuclease domain mutations in hypermutant tumors (31).

A disruption of DNA repair pathways will increase mutagenesis and genome instability, thereby affecting cancer progression and drug resistance (32). Here, we found that somatic SNVs in hypermutant tumors are mainly enriched in the p53 pathway. This observation may be linked to the high frequency of TP53 mutations. In addition, SNV and CNV frequency was found to be high in EGFR, KRAS and PIK3CA. Studies using new technologies such as liquid biopsy and nextgeneration sequencing have revealed that the mechanism of anti-
EGFR treatment resistance involves acquired mutations in the KRAS and EGFR ectodomain (33), and PIK3CA mutations are closely related to KRAS mutations (34). These data characterized tumors involving specific gene mutations.

\section{CONCLUSION}

In this study, we collected data on 5,980 tumor samples involving 23 types of solid tumors and performed a comprehensive analysis on the relationship between hypermutation and gene mutation, MSI, and PD-L1, as well as its clinical significance and 
A
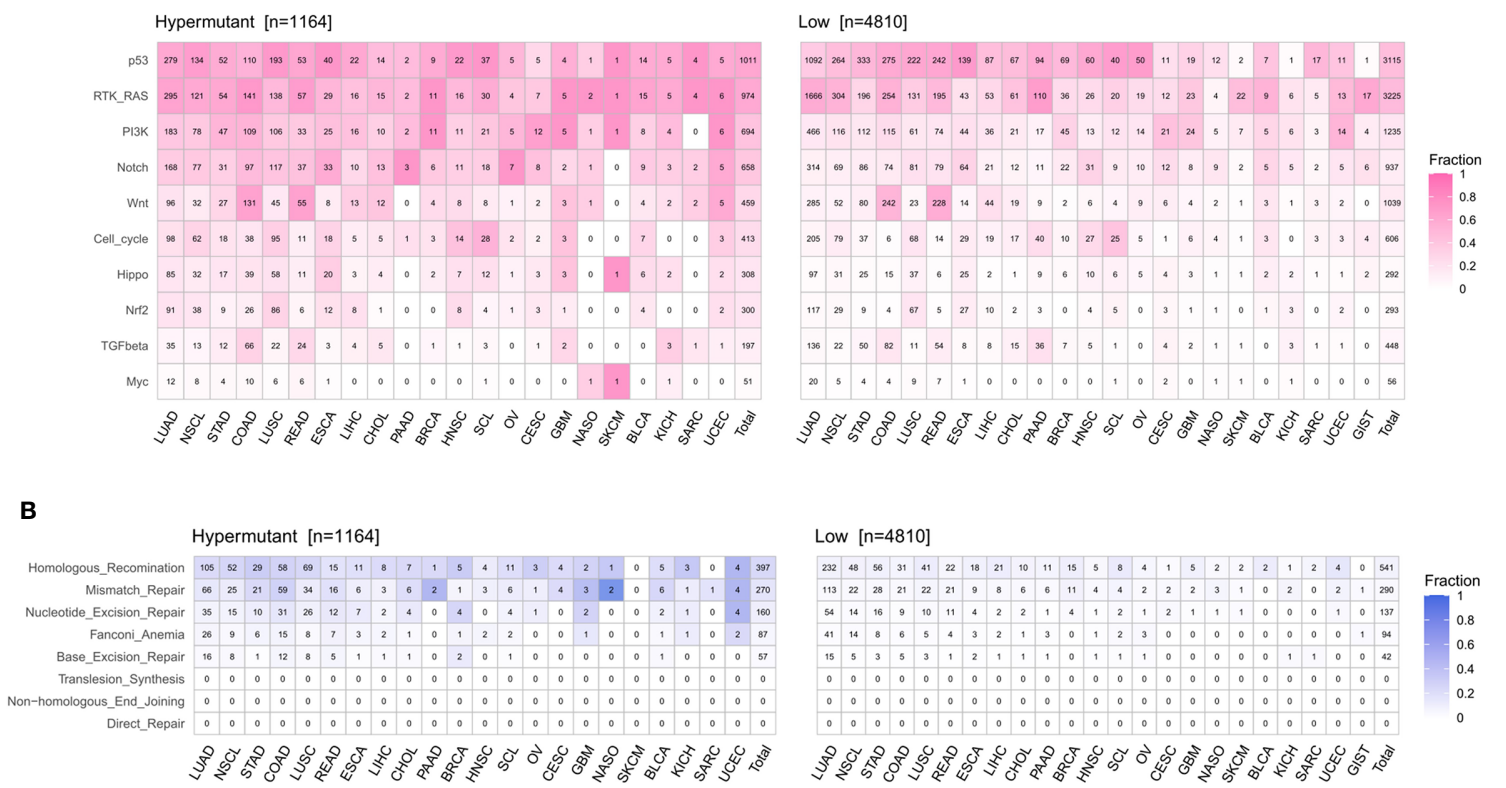

FIGURE 6 | The number and frequency of mutant samples in different pathways in Hypermutant and Low groups for each cancer type. (A) The oncogenic signaling pathways were illustrated in pink. (B) DNA damage repair pathways were depicted in blue.

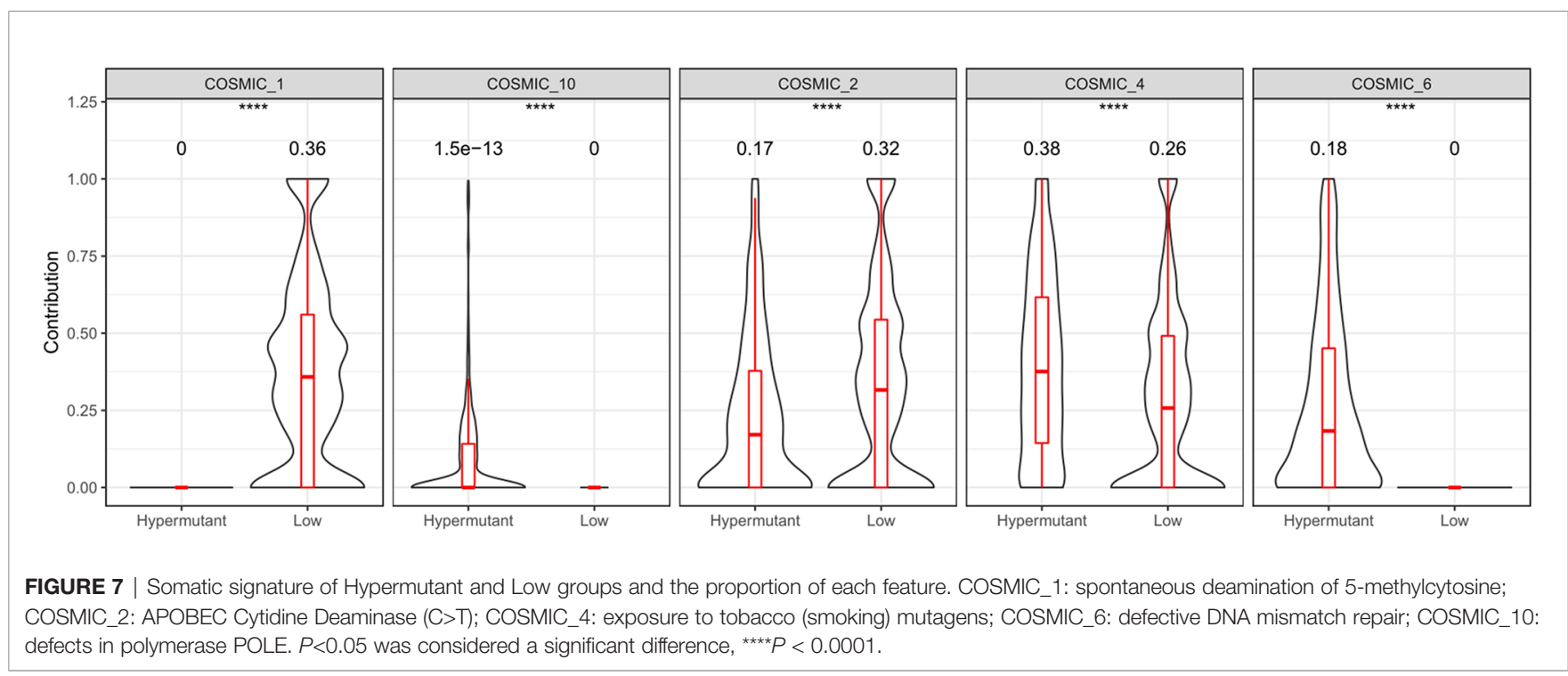

characterized the relationship between genotype and phenotype in hypermutant tumors. The overlap between high TMB and MSI-H or high PD-L1 is most likely attributable to MMR and/or POL mutations. In addition, hypermutant tumors displayed a higher rate of cancerous driver gene changes than tumors with non-hypermutation.

\section{DATA AVAILABILITY STATEMENT}

The datasets presented in this study can be found in online repositories. The names of the repository/repositories and accession number(s) can be found below: https://bigd.big.ac.cn/ bioproject/browse/PRJCA004800, PRJCA004800. 


\section{ETHICS STATEMENT}

The studies involving human participants were reviewed and approved by Ruijin Hospital. The patients/participants provided their written informed consent to participate in this study.

\section{AUTHOR CONTRIBUTIONS}

JZ conceived the study. HY, JJ, and JZ designed the study and wrote the draft manuscript. MS, YS, JLiu, and JW analyzed and interpreted the data. CY and WX collected and interpreted the clinical data. QL and WZ extracted the data and assess the data quality. JLi and XG did literature searches and identified the eligible studies. All authors contributed to the article and approved the submitted version.

\section{REFERENCES}

1. Hoadley KA, Yau C, Wolf DM, Cherniack AD, Tamborero D, Ng S, et al. Multiplatform Analysis of 12 Cancer Types Reveals Molecular Classification Within and Across Tissues of Origin. Cell (2014) 158(4):929-44. doi: 10.1016/ j.cell.2014.06.049

2. Tabassum N, Constantin TA, Cereser B, Stebbing J. A Cell-Cycle Signature Classifier for Pan-Cancer Analysis. Oncogene (2020) 39(38):6041-2. doi: 10.1038/s41388-020-01426-5

3. Palazzo M, Beauseroy P, Yankilevich P. A Pan-Cancer Somatic Mutation Embedding Using Autoencoders. BMC Bioinf (2019) 20(1):655. doi: 10.1186/ s12859-019-3298-z

4. Wang HL, Liu PF, Yue J, Jiang WH, Cui YL, Ren H, et al. Somatic Gene Mutation Signatures Predict Cancer Type and Prognosis in Multiple Cancers With Pan-Cancer 1000 Gene Panel. Cancer Lett (2020) 470:181-90. doi: 10.1016/j.canlet.2019.11.022

5. Benstead-Hume G, Wooller SK, Downs JA, Pearl FMG. Defining Signatures of Arm-Wise Copy Number Change and Their Associated Drivers in Kidney Cancers. Int J Mol Sci (2019) 20(22):5762. doi: 10.3390/ijms20225762

6. Wee Y, Wang T, Liu Y, Li X, Zhao M. A Pan-Cancer Study of Copy Number Gain and Up-Regulation in Human Oncogenes. Life Sci (2018) 211:206-14. doi: 10.1016/j.lfs.2018.09.032

7. Alexandrov LB, Kim J, Haradhvala NJ, Huang MN, Tian Ng AW, Wu Y, et al. The Repertoire of Mutational Signatures in Human Cancer. Nature (2020) 578(7793):94-101. doi: 10.1038/s41586-020-1943-3

8. Sha D, Jin Z, Budczies J, Kluck K, Stenzinger A, Sinicrope FA. Tumor Mutational Burden as a Predictive Biomarker in Solid Tumors. Cancer Discovery (2020) 10(12):1808-25. doi: 10.1158/2159-8290.CD-20-0522

9. High TMB Predicts Immunotherapy Benefit. Cancer Discov, Vol. 8. American Association for Cancer Research (AACR) (2018). p. 668. doi: 10.1158/21598290.CD-NB2018-048

10. Chalmers ZR, Connelly CF, Fabrizio D, Gay L, Ali SM, Ennis R, et al. Analysis of 100,000 Human Cancer Genomes Reveals the Landscape of Tumor Mutational Burden. Genome Med (2017) 9(1):34. doi: 10.1186/s13073-017-0424-2

11. Ready N, Hellmann MD, Awad MM, Otterson GA, Gutierrez M, Gainor JF, et al. First-Line Nivolumab Plus Ipilimumab in Advanced non-Small-Cell Lung Cancer (Checkmate 568): Outcomes by Programmed Death Ligand 1 and Tumor Mutational Burden as Biomarkers. J Clin Oncol (2019) 37 (12):992-1000. doi: 10.1200/JCO.18.01042

12. Ott PA, Bang YJ, Piha-Paul SA, Razak ARA, Bennouna J, Soria JC, et al. TCell-Inflamed Gene-Expression Profile, Programmed Death Ligand 1 Expression, and Tumor Mutational Burden Predict Efficacy in Patients Treated With Pembrolizumab Across 20 Cancers: KEYNOTE-028. J Clin Oncol (2019) 37(4):318-27. doi: 10.1200/JCO.2018.78.2276

13. Muller MF, Ibrahim AE, Arends MJ. Molecular Pathological Classification of Colorectal Cancer. Virchows Arch (2016) 469(2):125-34. doi: 10.1007/s00428016-1956-3

\section{FUNDING}

The study was supported by National Science Foundation of China (81972707), Shanghai Rising-Star Program (20QA1406200), Shanghai Municipal Commission of Health and Family Planning (20184Y0048), Guangci Distinguished Young Scholars Training Program (GCQN-2018-A06), and Shanghai Anticancer Association EYAS PROJECT (SACA-CY19C19).

\section{SUPPLEMENTARY MATERIAL}

The Supplementary Material for this article can be found online at: https://www.frontiersin.org/articles/10.3389/fonc.2021. 682017/full\#supplementary-material

14. Yuza K, Nagahashi M, Watanabe S, Takabe K, Wakai T. Hypermutation and Microsatellite Instability in Gastrointestinal Cancers. Oncotarget (2017) 8 (67):112103-15. doi: 10.18632/oncotarget.22783

15. Indraccolo S, Lombardi G, Fassan M, Pasqualini L, Giunco S, Marcato R, et al. Genetic, Epigenetic, and Immunologic Profiling of MMR-Deficient Relapsed Glioblastoma. Clin Cancer Res (2019) 25(6):1828-37. doi: 10.1158/10780432.CCR-18-1892

16. Hu H, Mu Q, Bao Z, Chen Y, Liu Y, Chen J, et al. Mutational Landscape of Secondary Glioblastoma Guides MET-Targeted Trial in Brain Tumor. Cell (2018) 175(6):1665-78.e18. doi: 10.1016/j.cell.2018.09.038

17. Ritch E, Fu SYF, Herberts C, Wang G, Warner EW, Schonlau E, et al. Identification of Hypermutation and Defective Mismatch Repair in Ctdna From Metastatic Prostate Cancer. Clin Cancer Res (2020) 26(5):1114-25. doi: 10.1158/1078-0432.CCR-19-1623

18. Daniel P, Sabri S, Chaddad A, Meehan B, Jean-Claude B, Rak J, et al. Temozolomide Induced Hypermutation in Glioma: Evolutionary Mechanisms and Therapeutic Opportunities. Front Oncol (2019) 9:41. doi: 10.3389/fonc. 2019.00041

19. Muggeo VM. Estimating Regression Models With Unknown Break-Points. Stat Med (2003) 22(19):3055-71. doi: 10.1002/sim.1545

20. Muggeo V. Segmented: An R Package to Fit Regression Models With BrokenLine Relationships. R News (2008) 8:20-5.

21. Yoshimura K, Inoue Y, Karayama M, Tsuchiya K, Mori K, Suzuki Y, et al. Heterogeneity Analysis of PD-L1 Expression and Copy Number Status in EBUS-TBNA Biopsy Specimens of non-Small Cell Lung Cancer: Comparative Assessment of Primary and Metastatic Sites. Lung Cancer (2019) 134:202-9. doi: 10.1016/j.lungcan. 2019.06.002

22. Gong J, Wang C, Lee PP, Chu P, Fakih M. Response to PD-1 Blockade in Microsatellite Stable Metastatic Colorectal Cancer Harboring a POLE Mutation. J Natl Compr Canc Netw (2017) 15(2):142-7. doi: 10.6004/ jnccn.2017.0016

23. Cristescu R, Mogg R, Ayers M, Albright A, Murphy E, Yearley J, et al. PanTumor Genomic Biomarkers for PD-1 Checkpoint Blockade-Based Immunotherapy. Science (2018) 362(6411):eaar3593. doi: 10.1126/ science.aar3593

24. Lee DW, Han SW, Bae JM, Jang H, Han H, Kim H, et al. Tumor Mutation Burden and Prognosis in Patients With Colorectal Cancer Treated With Adjuvant Fluoropyrimidine and Oxaliplatin. Clin Cancer Res (2019) 25 (20):6141-7. doi: 10.1158/1078-0432.CCR-19-1105

25. Reynolds IS, O'Connell E, Fichtner M, McNamara DA, Kay EW, Prehn JHM, et al. Mucinous Adenocarcinoma of the Colon and Rectum: A Genomic Analysis. J Surg Oncol (2019) 120(8):1427-35. doi: 10.1002/ jso. 25764

26. Strickland KC, Howitt BE, Shukla SA, Rodig S, Ritterhouse LL, Liu JF, et al. Association and Prognostic Significance of BRCA1/2-Mutation Status With Neoantigen Load, Number of Tumor-Infiltrating Lymphocytes and 
Expression of PD-1/PD-L1 in High Grade Serous Ovarian Cancer. Oncotarget (2016) 7(12):13587-98. doi: 10.18632/oncotarget.7277

27. De’ Angelis GL, Bottarelli L, Azzoni C, De’ Angelis N, Leandro G, Di Mario F, et al. Microsatellite Instability in Colorectal Cancer. Acta Biomed (2018) 89(9S):97-101. doi: 10.23750/abm.v89i9-S.7960

28. Llosa NJ, Cruise M, Tam A, Wicks EC, Hechenbleikner EM, Taube JM, et al. The Vigorous Immune Microenvironment of Microsatellite Instable Colon Cancer is Balanced by Multiple Counter-Inhibitory Checkpoints. Cancer Discovery (2015) 5 (1):43-51. doi: 10.1158/2159-8290.CD-14-0863

29. Le DT, Uram JN, Wang H, Bartlett BR, Kemberling H, Eyring AD, et al. PD-1 Blockade in Tumors With Mismatch-Repair Deficiency. N Engl J Med (2015) 372(26):2509-20. doi: 10.1056/NEJMoa1500596

30. Pardini B, Corrado A, Paolicchi E, Cugliari G, Berndt SI, Bezieau S, et al. DNA Repair and Cancer in Colon and Rectum: Novel Players in Genetic Susceptibility. Int J Cancer (2020) 146(2):363-72. doi: 10.1002/ijc.32516

31. Park VS, Pursell ZF. POLE Proofreading Defects: Contributions to Mutagenesis and Cancer. DNA Repair (Amst) (2019) 76:50-9. doi: 10.1016/ j.dnarep.2019.02.007

32. Chatterjee N, Walker GC. Mechanisms of DNA Damage, Repair, and Mutagenesis. Environ Mol Mutagen (2017) 58(5):235-63. doi: 10.1002/ em.22087
33. Miyamoto Y, Suyama K, Baba H. Recent Advances in Targeting the EGFR Signaling Pathway for the Treatment of Metastatic Colorectal Cancer. Int J Mol Sci (2017) 18(4):752. doi: 10.3390/ijms18040752

34. Jin J, Shi Y, Zhang S, Yang S. PIK3CA Mutation and Clinicopathological Features of Colorectal Cancer: A Systematic Review and Meta-Analysis. Acta Oncol (2020) 59(1):66-74. doi: 10.1080/0284186X.2019.1664764

Conflict of Interest: QL, WZ, JL and XG were employed by Genecast Biotechnology Co., Ltd.

The remaining authors declare that the research was conducted in the absence of any commercial or financial relationships that could be construed as a potential conflict of interest.

Copyright (C) 2021 Yuan, Ji, Shi, Shi, Liu, Wu, Yang, Xi, Li, Zhu, Li, Gong and Zhang. This is an open-access article distributed under the terms of the Creative Commons Attribution License (CC BY). The use, distribution or reproduction in other forums is permitted, provided the original author(s) and the copyright owner(s) are credited and that the original publication in this journal is cited, in accordance with accepted academic practice. No use, distribution or reproduction is permitted which does not comply with these terms. 